\title{
Comparison of CSF markers and semi-quantitative amyloid PET in Alzheimer's disease diagnosis and in cognitive impairment prognosis using the ADNI-2 database
}

Fayçal Ben Bouallègue ${ }^{1,2,3^{*}}$ (D), Denis Mariano-Goulart ${ }^{3}$, Pierre Payoux ${ }^{1,2}$ and the Alzheimer's Disease Neuroimaging Initiative (ADNI)

\begin{abstract}
Background: The relative performance of semi-quantitative amyloid positron emission tomography (PET) and cerebrospinal fluid (CSF) markers in diagnosing Alzheimer's disease (AD) and predicting the cognitive evolution of patients with mild cognitive impairment (MCI) is still debated.

Methods: Subjects from the Alzheimer's Disease Neuroimaging Initiative 2 with complete baseline cognitive assessment (Mini Mental State Examination, Clinical Dementia Rating [CDR] and Alzheimer's Disease Assessment Scale-Cognitive Subscale [ADAS-cog] scores), CSF collection (amyloid- $\beta_{1-42}[A B]$, tau and phosphorylated tau) and ${ }^{18}$ F-florbetapir scans were included in our cross-sectional cohort. Among these, patients with $\mathrm{MCl}$ or substantial memory complaints constituted our longitudinal cohort and were followed for $30 \pm 16$ months. PET amyloid deposition was quantified using relative retention indices (standardised uptake value ratio [SUVr]) with respect to pontine, cerebellar and composite reference regions. Diagnostic and prognostic performance based on PET and CSF was evaluated using ROC analysis, multivariate linear regression and survival analysis with the Cox proportional hazards model.

Results: The cross-sectional study included 677 participants and revealed that pontine and composite SUVr values were better classifiers (AUC 0.88, diagnostic accuracy 85\%) than CSF markers (AUC 0.83 and 0.85 , accuracy $80 \%$ and 75\%, for $A \beta$ and tau, respectively). SUVr was a strong independent determinant of cognition in multivariate regression, whereas $A \beta$ was not; tau was also a determinant, but to a lesser degree. Among the 396 patients from the longitudinal study, 82 (21\%) converted to AD within $22 \pm 13$ months. Optimal SUVr thresholds to differentiate AD converters were quite similar to those of the cross-sectional study. Composite SUVr was the best AD classifier (AUC 0.86, sensitivity 88\%, specificity 81\%). In multivariate regression, baseline cognition (CDR and ADAS-cog) was the main predictor of subsequent cognitive decline. Pontine and composite SUVr were moderate but independent predictors of final status and CDR/ADAS-cog progression rate, whereas baseline CSF markers had a marginal influence. The adjusted HRs for AD conversion were $3.8(p=0.01)$ for PET profile, $1.2(p=n s)$ for A $\beta$ profile and $1.8(p=0.03)$ for tau profile.
\end{abstract}

Conclusions: Semi-quantitative amyloid PET appears more powerful than CSF markers for AD grading and MCI prognosis in terms of cognitive decline and AD conversion.

Keywords: Alzheimer's disease, MCl, Amyloid PET, CSF markers, ADNI

\footnotetext{
* Correspondence: faybenb@hotmail.com

${ }^{1}$ Toulouse Neurolmaging Centre (ToNIC), Université de Toulouse, Inserm/UPS, Toulouse, France

${ }^{2}$ Nuclear Medicine Department, Purpan University Hospital, Toulouse, France

Full list of author information is available at the end of the article
} 


\section{Background}

Mild cognitive impairment (MCI) refers to cognitive deficits that do not directly impact the activities of daily living [1] and may be related to varied aetiologies, including depression, dementia and cerebrovascular disease. Only a small proportion of patients with MCI will convert to Alzheimer's disease (AD) within a given period of time, whereas the others will incur a variable cognitive decline or even revert to normal [2]. Considerable effort has been devoted to identifying and developing reliable biomarkers of incipient $\mathrm{AD}$ to target the individuals who would most benefit from early treatment intervention [3]. Decreased cerebrospinal fluid (CSF) concentration of the amyloid- $\beta_{1-42}$ peptide $(A \beta)$ and an increased level of the protein tau are seen in patients with $\mathrm{AD}[4,5]$. This pathological CSF signature is a key feature in AD diagnosis, and the CSF profile, potentially combined with neuroimaging data [6-9], has the ability to predict cognitive decline and conversion to AD independently of established risk factors such as age, sex and apolipoprotein E (ApoE) genotype [10-13].

Positron emission tomography (PET) using ${ }^{11} \mathrm{C}$-labelled Pittsburgh Compound B (PiB) or fluorinated tracers such as ${ }^{18}$ F-florbetapir allows in vivo visualisation and quantification of cortical $A \beta$ deposition with high sensitivity and specificity compared with amyloid plaque burden at autopsy $[14,15]$. Therefore, amyloid PET was included as a pathophysiological marker in the most recent international working group diagnostic criteria [16]. Although standard interpretation relies on visual assessment, semiquantitative measures of cortical retention with respect to a reference subcortical region is expected to provide refined evaluation of the amyloid burden with high testretest reliability [17]. Historically, normalisation of standardised uptake value (SUV) has been done using the brainstem, pons or whole cerebellum as the reference region. However, there is growing evidence that composite reference regions that include some subcortical white matter induce less temporal variability in sequential measurements, yielding higher accuracy in assessing subtle time changes and greater power to detect $A \beta$ accumulation [18-20]. Researchers in several studies have reported the capacity of amyloid PET using fluorinated tracers (either visual [21], semi-quantitative [22, 23] or both [24]) to provide prognostic insight regarding cognitive decline and conversion to $\mathrm{AD}$ in patients with $\mathrm{MCI}$, in line with previous evidence of the prognostic value of $\mathrm{PiB}$ PET [25-29]. A recent multi-centre study demonstrated the clinical impact of florbetapir PET in terms of diagnostic confidence and drug treatment [30].

Although CSF and PET measures of $\mathrm{A} \beta$ deposition are highly correlated [31-34], the comparative relevance of these two markers in discriminating patients with $A D$ and predicting cognitive outcome in patients with $\mathrm{MCI}$ is still under debate [35]. Hake et al. showed that CSF and PET profiles were both discriminant in classifying healthy control subjects and patients with MCI vs patients with AD [36]. Palqvist et al. found that the PET standardised uptake value ratio (SUVr) was associated with disease stage (cognition, memory and hippocampal volume) in patients with $\mathrm{MCI}$, whereas CSF markers were not [37]. In recent studies, researchers concluded that CSF analysis might detect $\mathrm{A} \beta$ deposition earlier than PET [38] and that reduced CSF A $\beta$ might relate more to early-stage $\mathrm{AD}$, whereas the amyloid load assessed by PET is indicative of disease progression [39].

Schreiber et al. demonstrated that baseline florbetapir PET, rated either visually or using a cerebellar SUVr, was predictive of conversion to $\mathrm{AD}$ in a large longitudinal cohort [24]. The prognostic value of the baseline PET profile with respect to subsequent cognitive evolution was also highlighted, consistent with prior results derived from a retrospective study [22]. Yet, the exact added diagnostic and prognostic value of amyloid PET semi-quantitative indices compared with CSF markers is still unclear, and, relatedly, the optimal reference region for SUVr computation remains to be defined. In the present study, we systematically compared baseline CSF markers and PET semi-quantitative indices in terms of diagnostic value regarding baseline cognitive status, as well as prognostic value in patients with MCI regarding cognitive decline and conversion to $\mathrm{AD}$. In addition, we evaluated the performance of the SUVr computed using various well-established subcortical reference regions.

\section{Methods}

\section{Subjects}

In this study, we used participant data from the Alzheimer's Disease Neuroimaging Initiative (ADNI), a multicentre project with approximately 50 medical centres and university sites across the United States and Canada [40]. The ADNI was launched in 2003 as a public-private partnership led by Principal Investigator Michael W. Weiner, MD. Its primary goal was to examine how brain imaging and other biomarkers can be used to measure the progression of MCI and early AD. Determination of sensitive and specific markers of very early $\mathrm{AD}$ progression is expected to help researchers and clinicians develop new treatments and monitor their effectiveness, as well as lessen the time and cost of clinical trials. A detailed description of the inclusion criteria can be found on the ADNI webpage (http://www.adni-info.org). Subjects were between 55 and 90 years old and willing and able to undergo all test procedures, including neuroimaging, and had agreed to undergo longitudinal follow-up.

Cognitively normal participants were the control subjects in the ADNI study. They showed no signs of depression, MCI or dementia. Participants with significant 
memory complaint (SMC) scored within the normal range for cognition but indicated concerns and exhibited slight forgetfulness. Early and late MCI participants reported an SMC either autonomously or via an informant or clinician. However, other cognitive domains showed no significant impairment, activities of daily living were preserved, and there were no signs of dementia. Participants with AD met the National Institute of Neurological and Communicative Disorders and Stroke/Alzheimer's Disease and Related Disorders Association criteria for probable $\mathrm{AD}[41,42]$.

Data were downloaded from the ADNI database (adni.loni.usc.edu) and included all subjects recruited in the ADNI-2 with complete available baseline data regarding cognitive assessment, CSF markers and PET A $\beta$ quantitation. Our cross-sectional sample was made up of 677 subjects ( 157 control subjects, 95 with SMC, 301 with MCI among whom 153 had early MCI and 148 had late $\mathrm{MCI}$, and 124 with $\mathrm{AD}$ at the time of the florbetapir scan; see Table 1) who were recruited between January 2011 and September 2013, and each had a baseline CSF collection and florbetapir session. The time delay between the lumbar puncture and the florbetapir PET was $11 \pm 18$ days. Our longitudinal sample was made up

Table 1 Baseline demographics, apolipoprotein E status and cerebrospinal fluid markers in the cross-sectional population by baseline status

\begin{tabular}{|c|c|c|c|}
\hline Baseline status & $\begin{array}{l}\text { Control subjects } \\
(n=157)\end{array}$ & $\begin{array}{l}\text { SMC/MCl } \\
(n=396)\end{array}$ & $\begin{array}{l}\mathrm{AD} \\
(n=124)\end{array}$ \\
\hline Male sex & 77 (49\%) & $204(52 \%)$ & $72(58 \%)$ \\
\hline Age, years & $74 \pm 6$ & $72 \pm 7^{\mathrm{a}}$ & $75 \pm 8$ \\
\hline ApoE4 carriers & $42(27 \%)$ & $186(47 \%)^{b}$ & $82(66 \%)^{c}$ \\
\hline \multicolumn{4}{|l|}{ Baseline cognition } \\
\hline GDS & $0.7 \pm 1.1$ & $1.4 \pm 1.4^{b}$ & $1.7 \pm 14^{c}$ \\
\hline MMSE & $29 \pm 1$ & $27 \pm 3^{b}$ & $23 \pm 2^{c}$ \\
\hline CDR 0 & 157 (100\%) & $96(24 \%)^{b}$ & $0^{c}$ \\
\hline CDR 0.5 & 0 & $299(76 \%)^{a}$ & $53(43 \%)^{c}$ \\
\hline$C D R \geq 1$ & 0 & $1(0.3 \%)$ & $71(57 \%)^{c}$ \\
\hline ADAS-cog & $9 \pm 5$ & $16 \pm 10^{b}$ & $31 \pm 8^{c}$ \\
\hline \multicolumn{4}{|l|}{ Baseline CSF } \\
\hline$A \beta_{1-42}, n g / L$ & $196 \pm 50$ & $175 \pm 53^{b}$ & $137 \pm 38^{c}$ \\
\hline Tau, ng/L & $67 \pm 34$ & $88 \pm 54^{b}$ & $133 \pm 65^{c}$ \\
\hline $\mathrm{p}-\mathrm{Tau}_{181}, \mathrm{ng} / \mathrm{L}$ & $33 \pm 16$ & $43 \pm 26^{b}$ & $60 \pm 35^{c}$ \\
\hline$p-\operatorname{Tau}_{181} / A \beta_{1-42}$ & $0.19 \pm 0.13$ & $0.29 \pm 0.24^{b}$ & $0.48 \pm 0.32^{c}$ \\
\hline \multicolumn{4}{|c|}{$\begin{array}{l}\text { Abbreviations: } A \beta \text { Amyloid- } \beta_{1-42}, A D \text { Alzheimer's disease, ADAS-cog Alzheimer's } \\
\text { Disease Assessment Scale-Cognitive Subscale, ApoE Apolipoprotein E, } \\
C D R \text { Clinical Dementia Rating, GDS Geriatric Depression Scale, MCI Mild } \\
\text { cognitive impairment, MMSE Mini Mental State Examination, } p \text {-tau Phosphorylated } \\
\text { tau, SMC Significant memory complaint } \\
{ }^{a} p<0.01 \text { vs control subjects } \\
{ }^{b} p<0.001 \text { vs control subjects } \\
c_{p}<0.001 \text { vs patients with } S M C / M C I\end{array}$} \\
\hline
\end{tabular}

of the 396 subjects with SMC and MCI from the crosssectional sample who had undergone an average clinical follow-up of $30 \pm 16$ months (see Table 2). Baseline visit and follow-up visits at 3, 6 and 12 months, then yearly, included complete cognitive assessment using the Geriatric Depression Scale, Mini Mental State Examination (MMSE), Clinical Dementia Rating (CDR) and Alzheimer's Disease Assessment Scale-Cognitive Subscale (ADAS-cog). Diagnostic status and cognitive scores were extracted from the latest available dataset ('DXSUM_PDXCONV_ ADNIALL.csv'). For each participant in the longitudinal cohort, the mean annual change in cognitive scores was computed by taking the difference between the last

Table 2 Baseline demographics, apolipoprotein E status, cerebrospinal fluid markers and clinical score evolution in the longitudinal cohort (patients with significant memory complaint/ mild cognitive impairment) by last known status

\begin{tabular}{|c|c|c|c|}
\hline Last known status & $\begin{array}{l}\text { Normal } \\
(n=105[27 \%])\end{array}$ & $\begin{array}{l}\mathrm{MCl} \\
(n=209[53 \%])\end{array}$ & $\begin{array}{l}\mathrm{AD} \\
(n=82[21 \%])\end{array}$ \\
\hline $\begin{array}{l}\text { Follow-up duration, } \\
\text { months }\end{array}$ & $25 \pm 12$ & $34 \pm 16^{a}$ & $36 \pm 13$ \\
\hline Male sex & $40(38 \%)$ & $121(58 \%)^{a}$ & $43(52 \%)$ \\
\hline Age, years & $71 \pm 6$ & $72 \pm 7$ & $73 \pm 7$ \\
\hline ApoE4 carriers & 41 (39\%) & $87(42 \%)$ & $58(71 \%)^{\mathrm{b}}$ \\
\hline \multicolumn{4}{|l|}{ Baseline cognition } \\
\hline GDS & $1.1 \pm 1.1$ & $1.8 \pm 1.4^{\mathrm{a}}$ & $1.8 \pm 14$ \\
\hline MMSE & $29 \pm 1$ & $28 \pm 2^{\mathrm{a}}$ & $27 \pm 2^{b}$ \\
\hline CDR 0 & $86(82 \%)$ & $9(4 \%)^{a}$ & $1(1 \%)^{b}$ \\
\hline CDR 0.5 & $19(18 \%)$ & $199(95 \%)^{a}$ & $81(99 \%)^{b}$ \\
\hline$C D R \geq 1$ & 0 & $1(0.5 \%)$ & 0 \\
\hline ADAS-cog & $9 \pm 4$ & $14 \pm 6^{\mathrm{a}}$ & $22 \pm 7^{\mathrm{b}}$ \\
\hline \multicolumn{4}{|l|}{ Baseline CSF } \\
\hline$A \beta_{1-42}(\mathrm{ng} / \mathrm{L})$ & $204 \pm 48$ & $179 \pm 53^{\mathrm{a}}$ & $141 \pm 35^{\mathrm{b}}$ \\
\hline Tau (ng/L) & $62 \pm 30$ & $77 \pm 44^{a}$ & $121 \pm 60^{b}$ \\
\hline $\mathrm{p}-\mathrm{Tau}_{181}(\mathrm{ng} / \mathrm{L})$ & $35 \pm 20$ & $38 \pm 23$ & $59 \pm 25^{b}$ \\
\hline$p-\operatorname{Tau}_{181} / A \beta_{1-42}$ & $0.19 \pm 0.15$ & $0.25 \pm 0.20^{c}$ & $0.44 \pm 0.21^{b}$ \\
\hline \multicolumn{4}{|l|}{ Follow-up } \\
\hline $\begin{array}{l}\text { MMSE annual } \\
\text { change }\end{array}$ & $-0.1 \pm 0.2$ & $-0.4 \pm 1.1$ & $-2 \pm 2.3^{b}$ \\
\hline CDR annual change & $0.01 \pm 0.14$ & $0.00 \pm 0.10$ & $0.23 \pm 0.21^{b}$ \\
\hline $\begin{array}{l}\text { ADAS-cog annual } \\
\text { change }\end{array}$ & $-0.1 \pm 2.3$ & $0.5 \pm 3.3$ & $3.7 \pm 3.8^{b}$ \\
\hline $\begin{array}{l}\text { Time to conversion, } \\
\text { months }\end{array}$ & - & - & $22 \pm 13$ \\
\hline
\end{tabular}

Abbreviations: $A \beta$ Amyloid- $\beta_{1-42}$, AD Alzheimer's disease, ADAS-cog Alzheimer's Disease Assessment Scale-Cognitive Subscale, ApoE Apolipoprotein E, CDR Clinical Dementia Rating, GDS Geriatric Depression Scale, MCI Mild cognitive impairment, MMSE Mini Mental State Examination, p-tau Phosphorylated tau, SMC Significant memory complaint

${ }^{a} p<0.001$ vs normal patients

${ }^{\mathrm{b}} p<0.001$ vs patients with $\mathrm{MC}$

${ }^{c} p<0.01$ vs normal patients 
cognitive evaluation and the baseline one and dividing by the time range. The last known diagnostic status was the one mentioned at the time of the last visit listed in the dataset. For each participant of the longitudinal cohort for whom the last status was $\mathrm{AD}$, time to conversion was computed as the delay between the baseline visit and the first visit mentioning an $\mathrm{AD}$ status.

\section{CSF markers}

Baseline $A \beta_{1-42}$, total tau and phosphorylated p-tau 181 (p-tau) were measured using the multiplex xMAP Luminex platform (Luminex Corp., Austin, TX, USA) with the INNO-BIA AlzBio3 kit (Innogenetics, Ghent, Belgium) $[5,43]$. For this study, we used the archived dataset 'UPENNBIOMK_MASTER.csv'. When multiple baseline CSF marker dosages were available, the median value was retained for subsequent analyses. The studied variables of CSF biomarker were $A \beta$, tau, $p$-tau and the $p$-tau/A $\beta$ ratio. Additional analysis details and quality control procedures appear on the ADNI website.

\section{Amyloid PET data}

Baseline $A \beta$ deposition was visualised using ${ }^{18}$ F-florbetapir PET. Semi-quantitative PET results were retrieved from the latest available dataset ('UCBERKELEYAV45_10_ 17_16.csv'). The methods for PET acquisition and analysis are described in more detail elsewhere [22, 44]. Florbetapir images consisted of $4 \times 5$-minute frames acquired at 50-70 minutes after injection, which were realigned, averaged, resliced to a common voxel size $(1.5 \mathrm{~mm})$ and smoothed to a common resolution of $8 \mathrm{~mm}$ in full width at halfmaximum [45]. Structural T1-weighted images acquired concurrently with the baseline florbetapir images were used as a structural template to define the cortical regions of interest and the reference regions in native space for each subject, using FreeSurfer (version 4.5.0; surfer.nmr.mgh.harvard.edu) as described elsewhere [44]. Baseline florbetapir scans for each subject were coregistered to baseline structural magnetic resonance imaging scans, which were subsequently used to extract weighted cortical retention indices (SUV) from grey matter within four large cortical regions of interest (frontal, cingulate, parietal and temporal cortices) that were averaged to create a mean cortical SUV as described in greater detail online (adni.bitbucket.org/docs/UCBERKE LEYAV45/UCBERKELEY_AV45_Methods_12.03.15.pdf). Cortical SUVr values were obtained by normalising cortical SUV with the mean uptake in a subcortical reference region. For the present study, candidate reference regions were pons, whole cerebellum and a composite region made up of the whole cerebellum, pons and eroded subcortical white matter [19]. In the sequel, the corresponding SUVr will be respectively referred to as pontine SUVr, cerebellar SUVr and composite SUVr.

\section{Statistical analyses}

Continuous variables are presented as mean \pm SD and categorical variables as number (percent). The diagnostic performance of CSF markers and SUVr was assessed through ROC analysis. For each parameter and each cutoff value, sensitivity was defined as the positivity rate in the patients with $\mathrm{AD}$ and specificity as the negativity rate in the control subjects/normal patients. The optimal cut-off value was that maximising Youden's index (sensitivity + specificity -1 ). The concordance between PET profile based on SUVr values and CSF profile was evaluated using Cohen's kappa coefficient.

To test the association of baseline SUVr and CSF markers with diagnosis and prognosis, a multivariate analysis was conducted using a stepwise linear regression model with an entry criterion of $p<0.05$ and a removal criterion of $p>0.1$. To identify the independent determinants of baseline status and baseline cognition (MMSE, CDR and ADAS-cog), the following explicative factors were included in the model: sex, age, ApoE4 status, the four CSF variables and SUVr. To identify the independent predictors of final status, cognitive decline (annual change in MMSE, CDR and ADAS-cog) and time to conversion, the following explicative factors were included in the model: sex, age, ApoE4 status, baseline cognitive scores, the four CSF variables and SUVr. Categorical variables (sex, ApoE4 status, baseline and final status) were discretised, whereas (pseudo-)continuous variables (age, cognitive scores, CSF markers and SUVr) were processed as such. In each model, the three SUVr values based on the three candidate reference regions were tested separately, then jointly.

The correlation between baseline SUVr and cognitive score evolution was evaluated using least-squares quadratic regression and Spearman's rank correlation. The statistical significance of the mean annual changes in cognitive scores was tested using a z-test.

The predictive value of baseline PET and CSF profiles regarding conversion to AD was assessed using KaplanMeier survival curves and the log-rank test. HRs were adjusted using a Cox proportional hazards model including the following explanatory covariates: sex, age, ApoE4 status, baseline cognitive scores, PET profile, CSF A $\beta$ and tau profiles. For patients who did not convert to $\mathrm{AD}$, survival data were considered censored from the time of the last visit on record.

A two-sided $p$ value $\leq 0.05$ was considered statistically significant. As regards the multivariate analysis, $p$ values were corrected for multiple comparisons using the Dunn-Šidák correction: $p_{\text {corrected }}=1-(1-p)^{m}$, with $m$ being the number of comparisons (here we set $m=9$ as the number of times the linear model was run). All statistical computations were performed using MATLAB R2013 software (MathWorks, Natick, MA, USA). 


\section{Results}

Figure 1 presents the patient flow diagram. For the cross-sectional cohort, the patient demographics, ApoE4 status, baseline cognitive scores and CSF markers are detailed in Table 1. The differences between control subjects and patients with MCI and between patients with MCI and patients with AD were highly significant for ApoE4 status, cognition and all four CSF markers. For the longitudinal cohort, the patient demographics, ApoE4 status, baseline cognition and CSF, annual change in cognitive scores during follow-up and time to conversion are detailed in Table 2. Of the 396 patients with SMC/MCI at baseline, 209 (53\%) were classified as having MCI at their last visit, 105 (27\%) were ranked as normal (mostly patients with baseline SMC, and 19 patients with baseline MCI who reverted to normal) and 82 (21\%) converted to AD (1 SMC, 19 early MCI and 62 late $\mathrm{MCI})$. The differences in baseline cognition and CSF markers were highly significant between normal subjects and patients with MCI and between patients with MCI and patients with AD. Cognitive decline was similar in normal subjects and patients with MCI and markedly greater in patients with AD.

Figure 2 shows the distribution of (from left to right) pontine, cerebellar and composite SUVr values in the cross-sectional and longitudinal cohorts. In both cohorts, SUVr values were significantly lower in normal

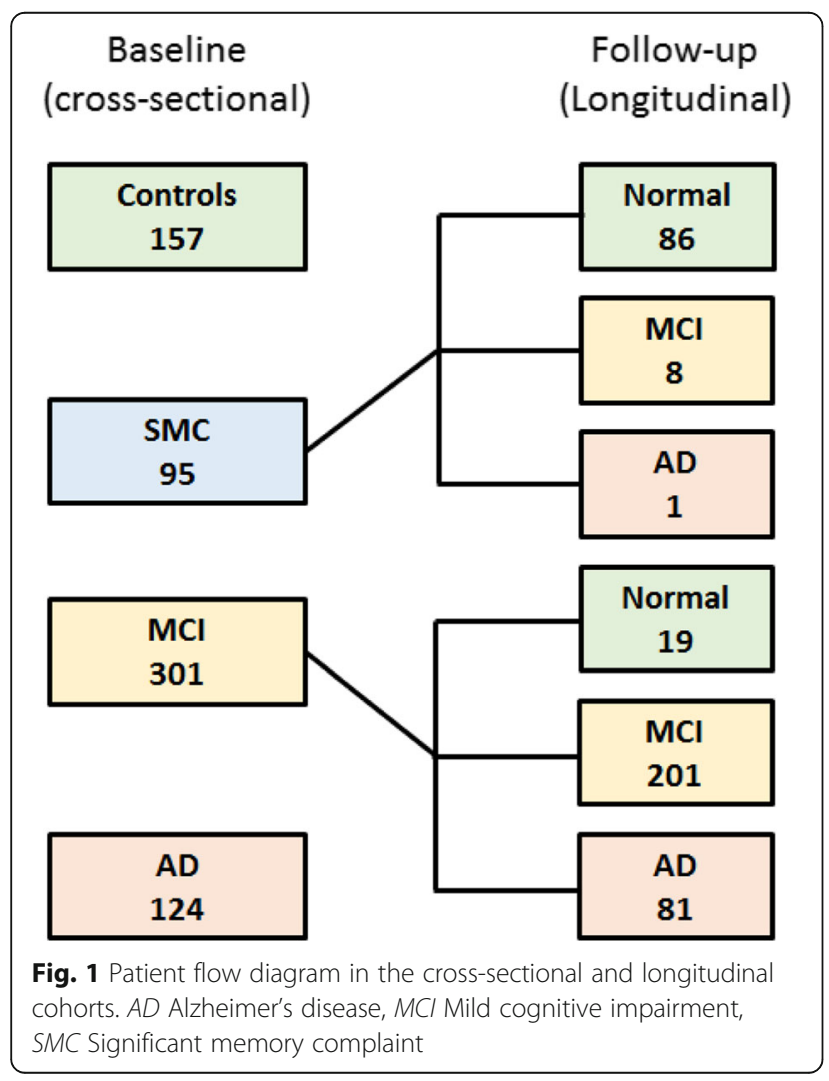

patients than in patients with MCI and in patients with MCI than in patients with $\mathrm{AD}$, whatever the reference region used. No difference was found between the homologous subsets of normal and patients with AD from the two cohorts.

Table 3 details the results of the ROC analyses for SUVr and CSF markers. Sensitivity and specificity stand for, respectively, the rate of true-positives among patients with $\mathrm{AD}$ and the rate of true-negatives among control subjects/normal patients. Optimal cut-off values for SUVr were highly similar in the cross-sectional and longitudinal cohorts, whereas they differed substantially for the CSF markers. SUVr performances were globally higher than those of CSF markers. In both cohorts, the best diagnostic performance was achieved using composite SUVr with an AUROC above 0.85, a sensitivity above $85 \%$ and a specificity above $80 \%$ in cross-sectional and longitudinal analyses. Overall, the predictive power of SUVr was superior to that of CSF markers, with risk ratios for evolving to $\mathrm{AD}$ ranging from 7 to 9.5 (vs 4.5 to 8 for CSF markers). Figure 3 shows the frequencies of final status in the longitudinal cohort according to baseline PET (composite SUVr) and baseline CSF profile (A $\beta /$ tau combination). Seventy-two percent of the patients had concordant $\mathrm{A} \beta /$ tau profiles (43\% negative, $29 \%$ positive), and $28 \%$ had discordant $A \beta /$ tau profiles $\left(25 \% \mathrm{~A} \beta^{+} / \mathrm{tau}^{-}\right.$and $\left.3 \% \mathrm{~A} \beta^{-} / \mathrm{tau}^{+}\right)$. There was no significant difference in mean follow-up duration between negative and positive profiles (PET, A $\beta$ or tau).

The concordance between the PET and CSF profiles was good when SUVr was compared with A $\beta$ (kappa > 0.8) and moderate when it was compared with tau and p-tau (kappa around $0.6-0.7$ ), without substantial variation related to the chosen reference region (see Table 4 for details).

Tables 5 and 6 summarise the results of the multivariate analyses. SUVr $p$ values reported in the tables are those obtained when the three SUVr values were evaluated separately. An asterisk designates the $p$ values that remained significant when the three SUVr values were evaluated jointly. The coefficients of determination $\left(r^{2}\right)$ reflect the proportion of the variance in the modelled variable that is predictable from each explanatory variable retained in the model. Regarding the cross-sectional cohort (Table 5), sex, tau level and SUVr were independent determinants of baseline status and cognitive scores (all corrected $p$ values <0.001), whereas ApoE4 status and other CSF variables were not. The best determinants were pontine and composite SUVr, which showed similarly high association with patient status and cognitive level. For the longitudinal cohort (Table 6), baseline cognition (MMSE, CDR and ADAS-cog) was the main predictor of cognitive decline in terms of final status and annual deterioration in cognitive scores. In patients with MCI who converted to AD during follow-up $(n=82)$, baseline ADAS-cog score 


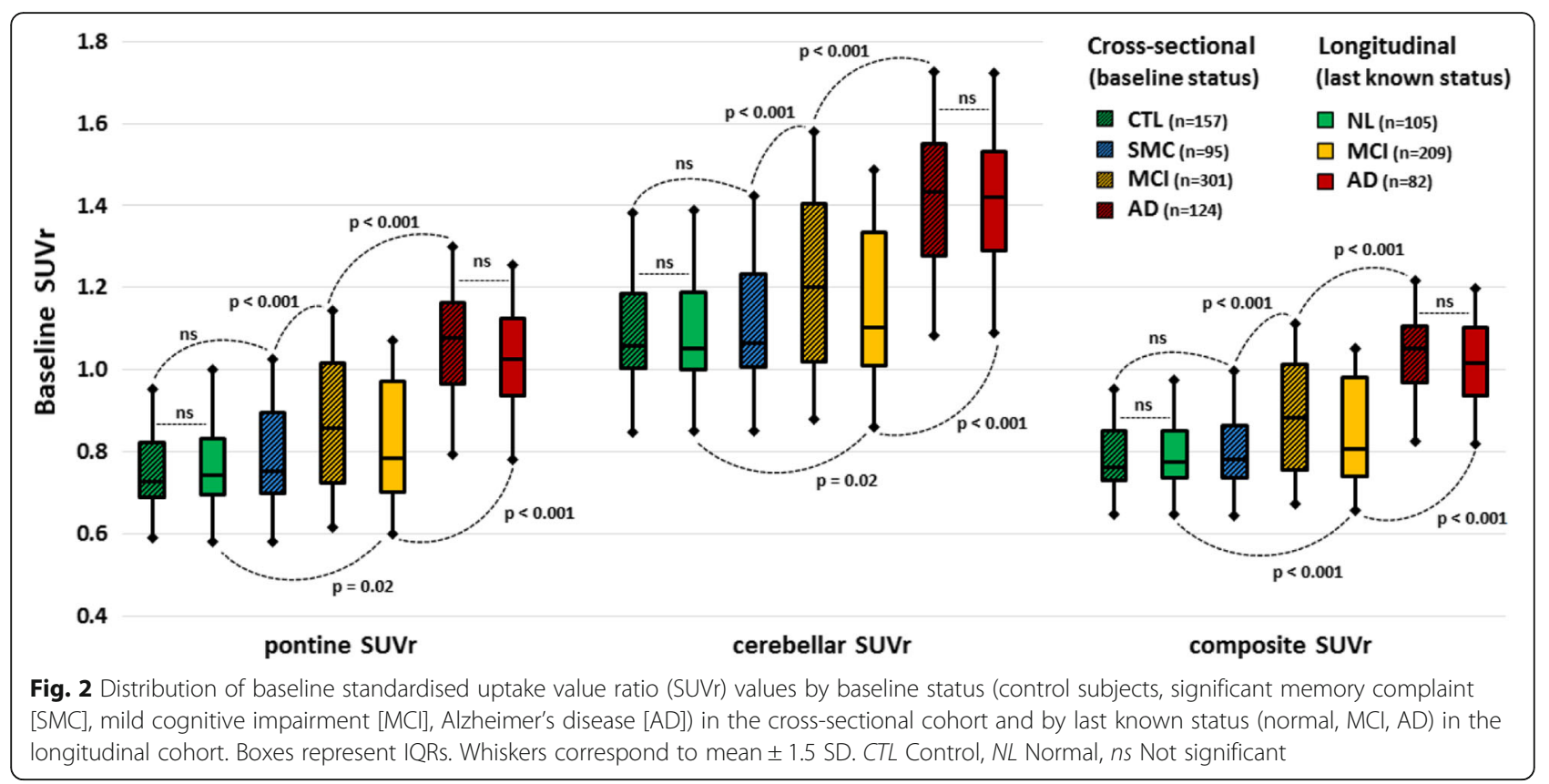

was the sole independent predictor of time to conversion $(p=0.03)$. CSF markers showed little or no association with cognitive evolution ( $\mathrm{p}$-tau on final status $p=0.02$, tau on MMSE change $p=0.02$ ). On the contrary, pontine and composite SUVr yielded significant additional prognostic information about final status $(p<0.001)$ and cognitive score decline $\left(p<10^{-5}\right.$ for CDR change, $p=0.001-0.005$ for ADAS-cog change).
The prognostic relevance of SUVr is further elaborated in Fig. 4. The scatterplots of the annual change in cognitive scores are displayed on top (Fig. 4a) according to the baseline composite SUVr, as are the associated quadratic regressions based on the whole longitudinal cohort. There was a moderate but significant Spearman's correlation of composite SUVr with cognitive decline $(r=0.33-0.37$, all $p$ values $<0.001$ ). The range and mean value (along with

Table 3 Results of ROC analyses for standardised uptake value ratio and cerebrospinal fluid markers

\begin{tabular}{|c|c|c|c|c|c|c|c|}
\hline & SUVr (pons) & SUVr (crb) & SUVr (comp) & $A \beta_{1-42}$ & Tau & $\mathrm{p}-\mathrm{Tau}_{181}$ & $p-T a u / A \beta$ \\
\hline \multicolumn{8}{|c|}{ Cross-sectional cohort } \\
\hline Optimal cut-off & 0.91 & 1.22 & 0.91 & 157 & 69 & 32 & 0.18 \\
\hline AUC & 0.88 & 0.84 & 0.88 & 0.83 & 0.85 & 0.79 & 0.85 \\
\hline Acc & $85 \%$ & $81 \%$ & $85 \%$ & $80 \%$ & $75 \%$ & $70 \%$ & $77 \%$ \\
\hline $\mathrm{Se}$ & $85 \%$ & $83 \%$ & $87 \%$ & $87 \%$ & $90 \%$ & $85 \%$ & $93 \%$ \\
\hline Sp & $85 \%$ & $79 \%$ & $83 \%$ & $75 \%$ & $63 \%$ & $59 \%$ & $65 \%$ \\
\hline \multicolumn{8}{|l|}{ Longitudinal cohort } \\
\hline Optimal cut-off & 0.91 & 1.24 & 0.89 & 171 & 88 & 45 & 0.27 \\
\hline AUC & 0.85 & 0.84 & 0.86 & 0.85 & 0.81 & 0.80 & 0.85 \\
\hline Acc & $82 \%$ & $82 \%$ & $84 \%$ & $79 \%$ & $79 \%$ & $75 \%$ & $82 \%$ \\
\hline $\mathrm{Se}$ & $83 \%$ & $84 \%$ & $88 \%$ & $90 \%$ & $68 \%$ & $71 \%$ & $83 \%$ \\
\hline Sp & $82 \%$ & $81 \%$ & $81 \%$ & $70 \%$ & $87 \%$ & $79 \%$ & $81 \%$ \\
\hline PPV & $43 \%$ & $44 \%$ & $42 \%$ & $35 \%$ & $44 \%$ & $43 \%$ & $44 \%$ \\
\hline NPV & $94 \%$ & $95 \%$ & $96 \%$ & $96 \%$ & $90 \%$ & $91 \%$ & $94 \%$ \\
\hline $\mathrm{RR}$ & 7.2 & 8.1 & 9.6 & 8.0 & 4.6 & 4.6 & 7.6 \\
\hline
\end{tabular}

Abbreviations: Acc Accuracy, Se Sensitivity (positivity rate among patients with Alzheimer's disease), Sp Specificity (negativity rate among control subjects/normal patients), PPV Positive predictive value, NPV Negative predictive value, RR Risk ratio for evolving to Alzheimer's disease, Pons Pontine, $c r b$ Cerebellar, 


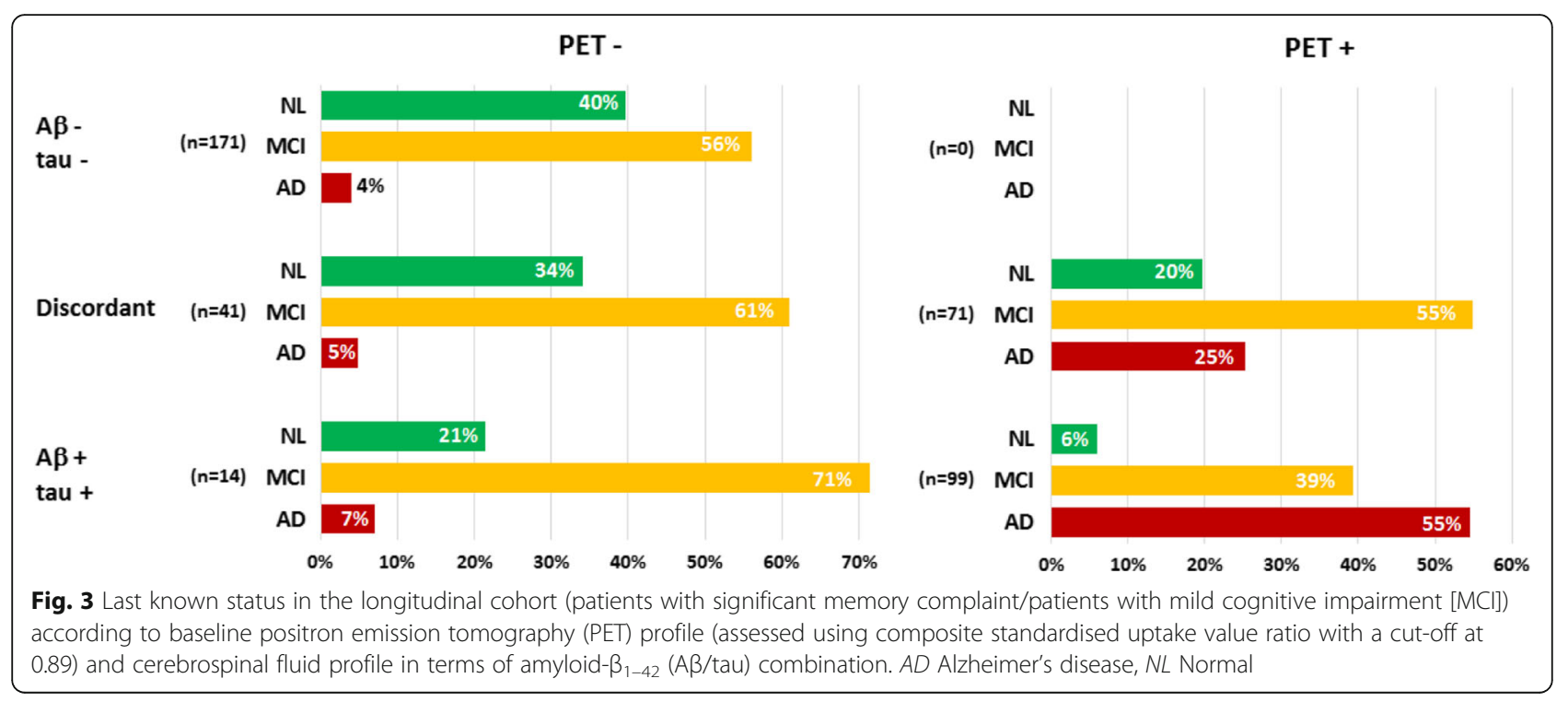

the $95 \% \mathrm{CI}$ ) of the score changes according to the baseline PET profile are shown in Fig. 4b. Patients with negative baseline PET incurred no significant change in CDR and ADAS-cog during follow-up. Conversely, patients with positive baseline PET exhibited clinically and statistically significant cognitive decline with mean annual changes of -1.2 in MMSE, +0.12 in CDR and +2.4 in ADAS-cog (all $p$ values $<0.001$ ).

Figure 5 presents the Kaplan-Meier curves for conversion to $\mathrm{AD}$ in patients with $\mathrm{SMC} / \mathrm{MCI}$ according to baseline PET (composite SUVr) and CSF profiles. The Cox proportional hazards model shows that baseline ADAS-cog score was the strongest predictor for AD conversion $\left(p<10^{-8}\right)$. A positive baseline PET was associated with an adjusted HR of 3.8 for AD conversion $(p=0.01)$. CSF A $\beta$ and tau were less predictive with adjusted HRs of 1.2 (not significant) and $1.8(p=0.03)$, respectively.

Table 4 Concordance (kappa) between positron emission tomography and cerebrospinal fluid profiles in the cross-sectional and longitudinal cohorts

\begin{tabular}{|c|c|c|c|c|}
\hline & $A \beta_{1-42}$ & Tau & $\mathrm{p}-\mathrm{Tau}_{181}$ & $p-T a u / A \beta$ \\
\hline \multicolumn{5}{|c|}{ Cross-sectional cohort } \\
\hline SUVr (pons) & 0.82 & 0.60 & 0.57 & 0.74 \\
\hline SUVr (crb) & 0.81 & 0.60 & 0.60 & 0.75 \\
\hline SUVr (comp) & 0.84 & 0.61 & 0.59 & 0.75 \\
\hline \multicolumn{5}{|c|}{ Longitudinal cohort } \\
\hline SUVr (pons) & 0.80 & 0.67 & 0.70 & 0.79 \\
\hline SUVr (crb) & 0.78 & 0.65 & 0.69 & 0.81 \\
\hline SUVr (comp) & 0.83 & 0.67 & 0.69 & 0.81 \\
\hline
\end{tabular}

Abbreviations: $A \beta$ Amyloid- $\beta_{1-42}, p$-tau Phosphorylated tau, SUVr Standardised uptake value ratio, Pons Pontine, crb Cerebellar, comp Composite

\section{Discussion}

In this study based on prospective data from the ADNI-2 cohort, we examined the complementary diagnostic and prognostic value of baseline CSF markers and ${ }^{18} \mathrm{~F}$-florbetapir $\mathrm{SUVr}$ values computed using three different reference regions. We found that PET semiquantitative assessment of $A \beta$ load was significantly superior, although CSF and PET markers were both relevant determinants of cognitive status and predictive of cognition decline in patients with MCI. Notably, as can be seen in Fig. 2 and Table 3, baseline SUVr distribution was similar in patients with baseline $\mathrm{AD}$ and patients with SMC/MCI who converted to $\mathrm{AD}$ during follow-up; hence, the optimal SUVr cut-offs to differentiate patients with AD from normal subjects were nearly identical in the crosssectional and longitudinal cohorts. The optimal cut-offs for CSF markers were less robust, suggesting that PET quantitation might be preferable for accurate selection and therapeutic monitoring of individuals in clinical trials [46].

Our optimal cerebellar SUVr cut-off (1.22) was consistent with that proposed by Fleisher et al. (1.17), based on post-mortem neuropathological data [47]. A less conservative SUVr cut-off was proposed by Joshi et al. [17] as the upper bound of a one-tailed $95 \% \mathrm{CI}$ of cerebellar SUVr distribution in young healthy control subjects, and it was used in other studies [22, 24] as a positivity threshold for florbetapir PET. Such a low threshold based on young control subjects seems questionable, however, and may result in poor specificity (about 70\% in the study by Landau et al. [22]), given that significant amyloid deposition without cognitive impairment is seen in $20 \%$ to $40 \%$ of normal elderly volunteers $[14,48]$. To our knowledge, this is the first attempt to provide optimal thresholds for pontine and composite SUVr, 
Table 5 Results of the stepwise linear regression investigating the association of baseline demographics, cerebrospinal fluid markers and positron emission tomography data, with baseline status and cognitive scores in the cross-sectional cohort

\begin{tabular}{|c|c|c|c|c|c|c|c|c|}
\hline \multirow[t]{2}{*}{ Association with } & \multicolumn{2}{|c|}{ Baseline status (CTL, SMC/MCl, AD) } & \multicolumn{2}{|c|}{ Baseline MMSE } & \multicolumn{2}{|c|}{ Baseline CDR } & \multicolumn{2}{|c|}{ Baseline ADAS-cog } \\
\hline & $r^{2}$ & $p$ Value & $\overline{r^{2}}$ & $p$ Value & $\overline{r^{2}}$ & $p$ Value & $r^{2}$ & $p$ Value \\
\hline$\overline{\text { Sex }}$ & 0.04 & $<0.001$ & 0.04 & $<10^{-4}$ & 0.04 & $<0.001$ & 0.08 & $<10^{-8}$ \\
\hline Age & & ns & 0.03 & 0.003 & & ns & 0.04 & $<0.001$ \\
\hline ApoE4 & & ns & & ns & & ns & & ns \\
\hline \multicolumn{9}{|l|}{ Baseline CSF } \\
\hline$A \beta_{1-42}$ & & ns & & ns & & ns & & ns \\
\hline Tau & 0.06 & $<10^{-5}$ & 0.10 & $<10^{-9}$ & 0.07 & $<10^{-7}$ & 0.12 & $<10^{-11}$ \\
\hline $\mathrm{p}-\mathrm{Tau}_{181}$ & & ns & & ns & & ns & & ns \\
\hline$p-T a u / A \beta$ & & ns & & ns & & ns & & ns \\
\hline \multicolumn{9}{|l|}{ Baseline PET } \\
\hline SUVr (pons) & 0.18 & $<10^{-19(*)}$ & 0.14 & $<10^{-15}$ & 0.14 & $<10^{-15}$ & 0.18 & $<10^{-24}$ \\
\hline SUVr (crb) & 0.10 & $<10^{-6(*)}$ & 0.02 & $0.03^{(*)}$ & & ns & 0.05 & $0.001^{(*)}$ \\
\hline SUVr (comp) & 0.17 & $<10^{-17}$ & 0.14 & $<10^{-14\left(^{*}\right)}$ & 0.14 & $<10^{-14\left(^{*}\right)}$ & 0.19 & $<10^{-23(*)}$ \\
\hline
\end{tabular}

Abbreviations: AD Alzheimer's disease, ADAS-cog Alzheimer's Disease Assessment Scale-Cognitive Subscale, ApoE Apolipoprotein E, CDR Clinical Dementia Rating, Comp Composite, Crb Cerebellar, CSF Cerebrospinal fluid, MCI Mild cognitive impairment, MMSE Mini Mental State Examination, Pons Pontine, SMC Significant memory complaint, SUVr Standardised uptake value ratio, ns Not significant

All $p$ values are corrected for multiple comparisons

(*): Remained an independent determinant when evaluated jointly

Table 6 Results of the stepwise linear regression investigating the association of baseline demographics, cognitive scores, cerebrospinal fluid markers and positron emission tomography data, with prognosis in terms of final status, cognitive score evolution and time to conversion in the longitudinal cohort

\begin{tabular}{|c|c|c|c|c|c|c|c|c|c|c|}
\hline \multirow[t]{2}{*}{ Association with } & \multicolumn{2}{|c|}{ Last known status (NL, MCl, AD) } & \multicolumn{2}{|c|}{ MMS annual change } & \multicolumn{2}{|c|}{ CDR annual change } & \multicolumn{2}{|c|}{ ADAS-cog annual change } & \multicolumn{2}{|c|}{ Time to conversion } \\
\hline & $\overline{r^{2}}$ & $p$ Value & $r^{2}$ & $p$ Value & $\overline{r^{2}}$ & $p$ Value & $\overline{r^{2}}$ & $p$ Value & $r^{2}$ & $p$ Value \\
\hline Sex & & ns & & ns & & ns & & ns & & ns \\
\hline Age & & ns & & ns & & ns & & ns & & ns \\
\hline ApoE4 & & ns & & ns & & ns & & ns & & ns \\
\hline \multicolumn{11}{|c|}{ Baseline cognition } \\
\hline GDS & & ns & & ns & 0.02 & 0.04 & & ns & & ns \\
\hline MMSE & & ns & 0.10 & $<10^{-8}$ & & ns & 0.10 & $<0.001$ & & ns \\
\hline CDR & 0.38 & $<10^{-16}$ & & ns & 0.08 & $<10^{-8}$ & & ns & & ns \\
\hline ADAS-cog & 0.18 & $<10^{-15}$ & 0.18 & $<10^{-18}$ & 0.14 & $<10^{-16}$ & & ns & 0.10 & 0.03 \\
\hline \multicolumn{11}{|l|}{ Baseline CSF } \\
\hline$A \beta_{1-42}$ & & ns & & ns & & ns & & ns & & ns \\
\hline Tau & & ns & 0.03 & 0.02 & & ns & & ns & & ns \\
\hline $\mathrm{p}-\mathrm{Tau}_{181}$ & 0.03 & 0.02 & & ns & & ns & & ns & & ns \\
\hline$p-T a u / A \beta$ & & ns & & ns & & ns & & ns & & ns \\
\hline \multicolumn{11}{|l|}{ Baseline PET } \\
\hline SUVr (pons) & 0.05 & $<0.001^{\mathrm{a}}$ & & ns & 0.06 & $<10^{-5 a}$ & 0.08 & $0.001^{a}$ & & ns \\
\hline SUVr (crb) & 0.03 & 0.02 & & ns & & ns & & ns & & ns \\
\hline SUVr (comp) & 0.05 & $<0.001$ & & ns & 0.05 & $<10^{-5}$ & 0.07 & 0.005 & & ns \\
\hline
\end{tabular}

Abbreviations: $A \beta$ Amyloid- $\beta_{1-42}$, AD Alzheimer's disease, ADAS-cog Alzheimer's Disease Assessment Scale-Cognitive Subscale, ApoE Apolipoprotein E, CDR Clinical Dementia Rating, Comp Composite, Crb Cerebellar, CSF Cerebrospinal fluid, GDS Geriatric Depression Scale, MCI Mild cognitive impairment, MMSE Mini Mental State Examination, NL Normal, PET Positron emission tomography, Pons Pontine, $p$-tau Phosphorylated tau, SUVr Standardised uptake value ratio, ns Not significant All $p$ values are corrected for multiple comparisons

${ }^{\text {a }}$ Remained an independent predictor when evaluated jointly 


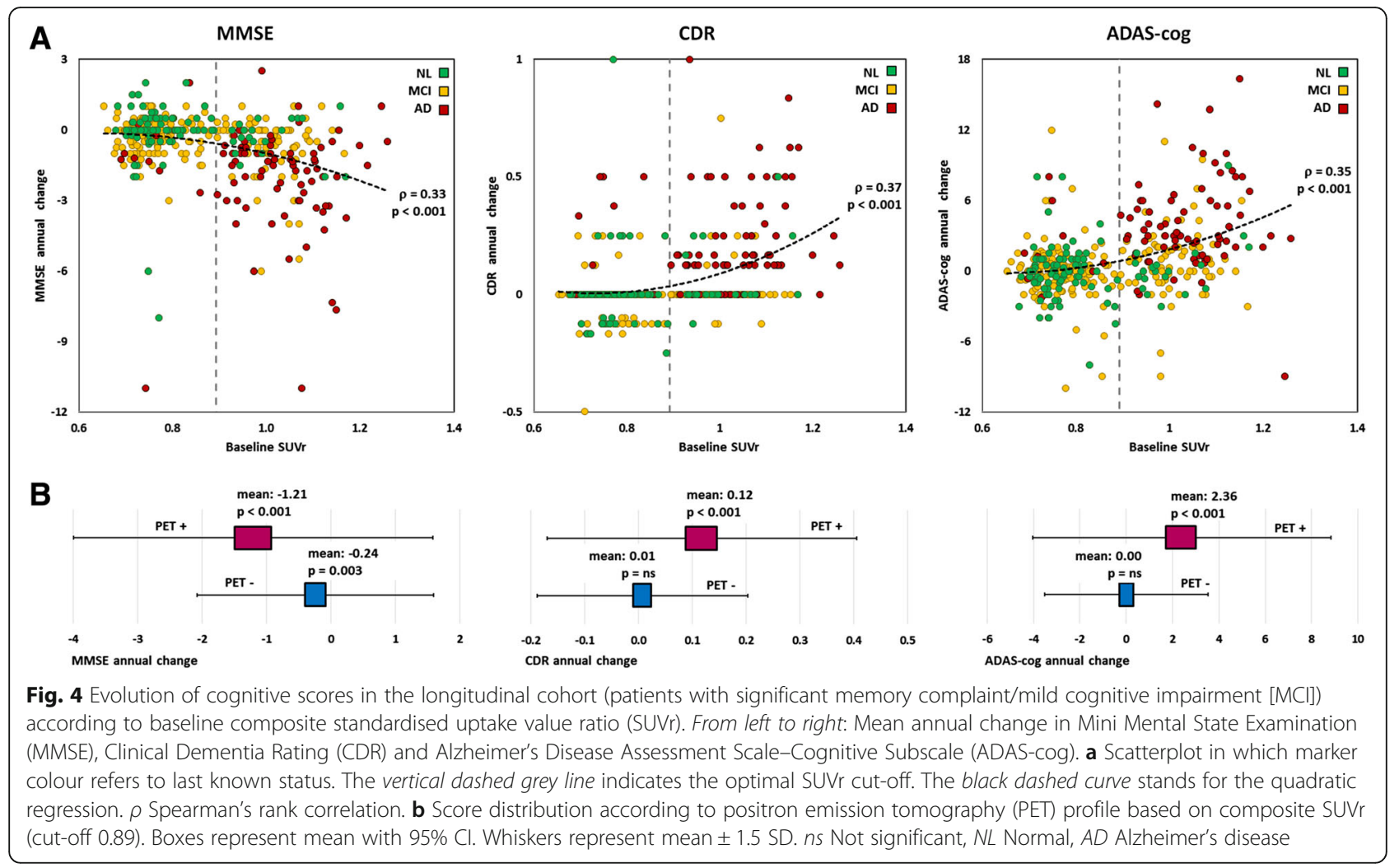

because recent studies involving extra-cerebellar reference regions have been aimed primarily at assessing the longitudinal accuracy of SUVr estimates [18, 19].

Regarding the CSF ROC analyses, our optimal A $\beta$ cutoff to differentiate patients with $\mathrm{AD}$ from normal control subjects $(157 \mathrm{ng} / \mathrm{L})$ was similar to that obtained by De Meyer et al. (159 ng/L) based on the ADNI-1 cohort [12]. Our optimal CSF A $\beta$ cut-off to predict conversion to $\mathrm{AD}$ in the longitudinal analysis $(171 \mathrm{ng} / \mathrm{L})$ was closest to that proposed by Shaw et al. (192 $\mathrm{ng} / \mathrm{L}$ ) with reference to

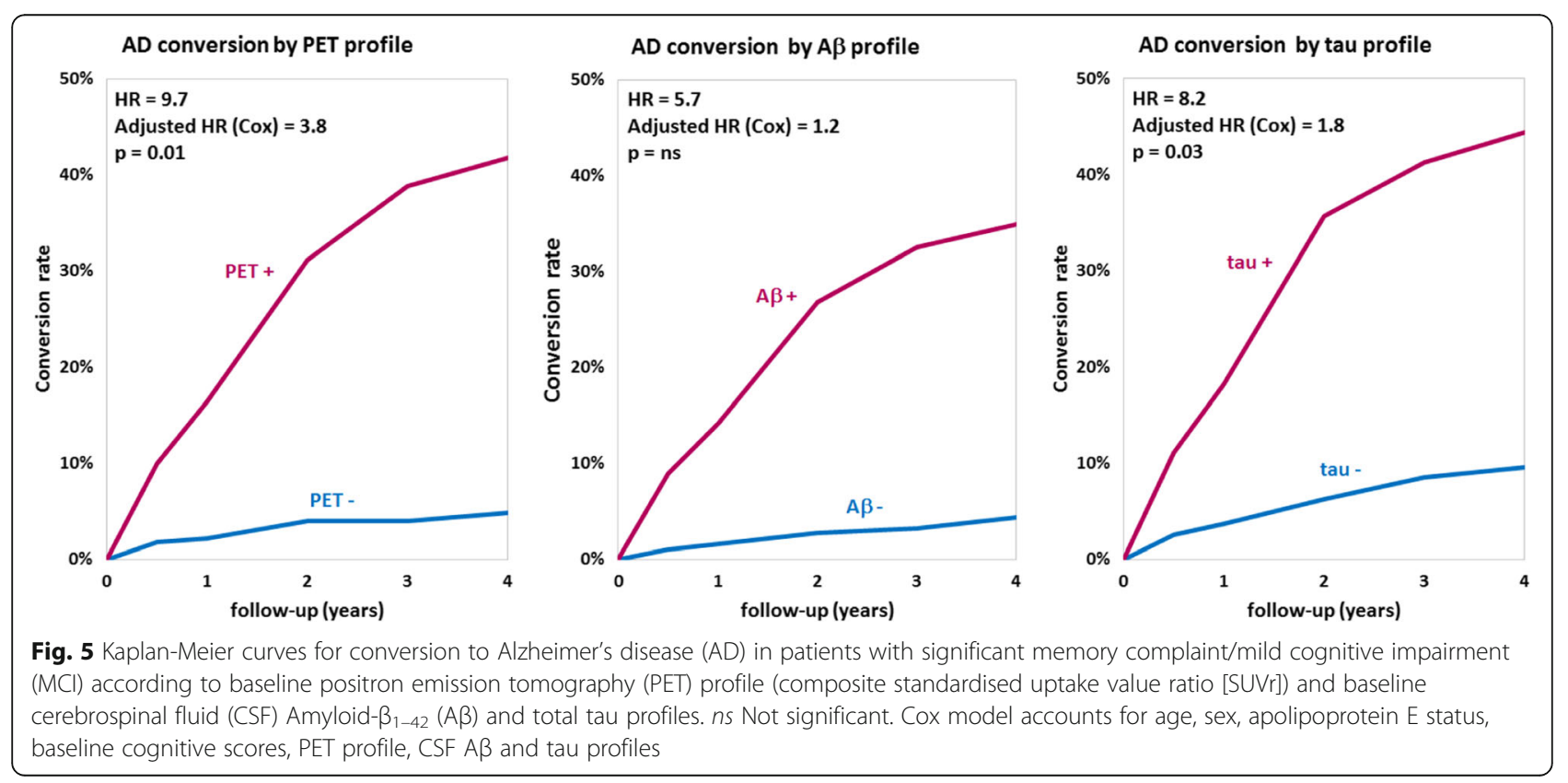


autopsy data [5], yielding comparable sensitivity and negative predictive value (respectively, 90\% and 96\% vs 96\% and 95\%). Our optimal cut-off for CSF tau (88 ng/L) was also similar to that mentioned by Shaw (93 ng/L) [5]. The proportion of concordant CSF profiles in terms of $A \beta$ and tau was $72 \%$ in both cohorts, concordant with the $73 \%$ of concordant profiles reported by Sunderland et al. [4] in a cohort of patients with $\mathrm{AD}$ and control subjects.

In our cross-sectional cohort, the first interesting finding was that PET SUVr clearly outperformed CSF markers in determining patients' cognitive status, as evaluated in a multivariate model. Its diagnostic accuracy neighboured $85 \%$ in differentiating patients with $\mathrm{AD}$ from control subjects, and cognitive performance (MMSE, CDR and ADAS-cog) was significantly associated with pontine and composite SUVr in the whole population. The higher diagnostic performance of pontine and composite SUVr than cerebellar SUVr might be related to a lower signal-to-noise ratio in the cerebellum, leading to less accurate and more variable SUV measurements in this region. Researchers in previous studies pointed out that pontine and cerebellar uptake was prone to noise and longitudinal variability owing to the small size of the considered regions and their peripheral location in the PET scanner field of view, and they advocated for the use of composite reference regions taking into account cerebral white matter $[18,19]$.

The CSF markers showed lower diagnostic value in ROC analysis (lower AUC and lower accuracy of $80 \%$ for $\mathrm{A} \beta$ and $75 \%$ for tau), and total tau was the sole CSF marker to bring added diagnostic value. Palmqvist et al. [35] noted that ${ }^{18}$ F-flutemetamol cerebellar SUVr was correlated with global cognition and hippocampal atrophy in patients with increased $A \beta$ load, whereas CSF $A \beta$ was not. These data are consistent with a commonly accepted model of $\mathrm{AD}$ pathological cascade, according to which $A \beta$ deposition takes place at an early stage in the natural history of the disease and tau-mediated neuronal injury occurs secondarily [3]. Yet, although CSF A $\beta$ reaches a plateau prior to the prodromal state, PET retention gradually increases during progression to $\mathrm{AD}$ [49]. Semi-quantitative amyloid PET may thus be more appropriate than CSF markers for early-stage grading of AD. To be fully operative and allow efficient discrimination between neurodegenerative diseases, it has to be integrated within the range of available biomarkers, including tau-specific PET tracers currently under clinical assessment [50].

The second original finding, which might have stronger practical implications, was that baseline PET SUVr was more predictive of clinical evolution and AD conversion than CSF markers and that baseline SUVr levels directly correlated with the subsequent rate of cognitive decline. Composite SUVr predictive accuracy regarding final status reached $84 \%$ compared with $79 \%$ for both CSF $A \beta$ and tau. In line with prior reports, cognitive measures at baseline were the best predictors of cognitive evolution and $\mathrm{AD}$ conversion [51, 52]. Baseline pontine and composite SUVr were moderate but significant predictors of final status and mean annual CDR and ADAS-cog change in multivariate analysis, whereas CSF markers had little or no impact on cognitive evolution. Cognitive decline as reflected by the mean annual changes in MMSE, CDR and ADAS-cog was significantly correlated with baseline composite SUVr. The mean annual changes in CDR and ADAS-cog were significant in patients with positive baseline PET, whereas patients with negative baseline PET did not incur significant CDR and ADAS-cog modification during follow-up (Fig. 4). Among patients with a negative baseline PET (rated using composite SUVr), 4\% were AD converters, and among those with a positive PET scan, $42 \%$ were $\mathrm{AD}$ converters. This yielded an adjusted $\mathrm{HR}$ for $\mathrm{AD}$ conversion of $3.8(p=0.01)$. Notably, the PET profile appeared decisive in patient with discordant CSF markers (99 $\mathrm{A} \beta^{+} / \mathrm{tau}^{-}$and $\left.13 \mathrm{~A} \beta^{-} / \mathrm{tau}^{+}\right)$. In these patients, an abnormal amyloid PET resulted in a five-fold increase in $\mathrm{AD}$ conversion risk ( $25 \%$ vs $5 \%$ in patients with a normal amyloid PET; see Fig. 3). It would seem that even in patients with a concordant positive CSF profile $\left(\mathrm{A} \beta^{+} / \mathrm{tau}^{+}\right)$, a negative $\mathrm{PET}$ is associated with a moderate risk of $\mathrm{AD}$ conversion (7\% vs $55 \%$ in patients with a positive PET), though $\mathrm{A}^{+} / \mathrm{tau}^{+} / \mathrm{PET}^{-}$profiles were too few to ensure sufficient statistical power. Patients with a negative PET profile who evolved to AD during follow-up might either correspond to PET false-negatives or to cases of non-amyloid dementias. The proportion of PET-positive patients who were ranked as normal during follow-up is consistent with previous evidence that $20 \%$ to $30 \%$ of cognitively normal elderly subjects harbour $A \beta$ deposition [53].

\section{Conclusions}

Semi-quantitative amyloid PET and CSF markers yield complementary information for classifying normal subjects, patients with $\mathrm{MCI}$ and patients with $\mathrm{AD}$. However, PET might be preferable for robust grading of early-stage $\mathrm{AD}$, and cross-sectional cut-off values for SUVr seem to be directly transposable for longitudinal analysis. Amyloid PET quantification using a composite SUVr appears more powerful than CSF markers for MCI prognosis in terms of $\mathrm{AD}$ conversion, and progressive cognitive decline is correlated with baseline composite SUVr. In patients with an equivocal CSF profile, amyloid PET effectively differentiates patients with high risk of AD conversion.

\section{Abbreviations}

Aß: Amyloid- $\beta_{1-42}$; Acc: Accuracy; AD: Alzheimer's disease; ADAS-cog: Alzheimer's Disease Assessment Scale-Cognitive Subscale; ADNI: Alzheimer's Disease Neuroimaging Initiative; ApoE: Apolipoprotein E; CDR: Clinical Dementia Rating; Comp: Composite; Crb: Cerebellar; CSF: Cerebrospinal fluid; GDS: Geriatric 
Depression Scale; MCl: Mild cognitive impairment; MMSE: Mini Mental State Examination; NPV: Negative predictive value; PET: Positron emission tomography; PiB: Pittsburgh Compound B; Pons: Pontine; PPV: Positive predictive value; RR: Risk ratio; Se: Sensitivity; SMC: Significant memory complaint; Sp: Specificity; SUV: Standardised uptake value; SUVr: Standardised uptake value ratio

\section{Acknowledgements}

Data used in preparation of this paper were obtained from the Alzheimer's Disease Neuroimaging Initiative (ADNI) database (http://adni.loni.usc.edu/). As such, the investigators within the ADNI contributed to the design and implementation of ADNI and/or provided data but did not participate in the analysis or in the writing of this paper. A complete listing of ADNI investigators can be found at http://adni.loni.usc.edu/wp-content/uploads/how_to_apply/ ADNI_Acknowledgement_List.pdf.

\section{Funding}

Data collection and sharing for this project were funded by the Alzheimer's Disease Neuroimaging Initiative (ADNI) (National Institutes of Health grant U01 AG024904) and U.S. Department of Defense ADNI (award number W81XWH-12-2-0012). ADNI is funded by the National Institute on Aging, the National Institute of Biomedical Imaging and Bioengineering, and through generous contributions from the following: AbbVie; Alzheimer's Association; Alzheimer's Drug Discovery Foundation; Araclon Biotech; BioClinica, Inc.; Biogen; Bristol-Myers Squibb Company; CereSpir, Inc.; Cogstate; Eisai Inc.; Elan Pharmaceuticals, Inc.; Eli Lilly and Company; EUROIMMUN; F. Hoffmann-La Roche Ltd and its affiliated company Genentech, Inc.; Fujirebio; GE Healthcare; IXICO Ltd.; Janssen Alzheimer Immunotherapy Research \& Development, LLC; Johnson \& Johnson Pharmaceutical Research \& Development LLC; Lumosity; Lundbeck; Merck \& Co., Inc;; Meso Scale Diagnostics, LLC; NeuroRx Research; Neurotrack Technologies; Novartis Pharmaceuticals Corporation; Pfizer Inc.; Piramal Imaging; Servier; Takeda Pharmaceutical Company; and Transition Therapeutics. The Canadian Institutes of Health Research is providing funds to support ADNI clinical sites in Canada. Private sector contributions are facilitated by the Foundation for the National Institutes of Health (www.fnih.org). The grantee organisation is the Northern California Institute for Research and Education, and the study is coordinated by the Alzheimer's Therapeutic Research Institute at the University of Southern California. ADNI data are disseminated by the Laboratory of Neuro Imaging at the University of Southern California.

\section{Availability of data and materials}

Not applicable.

\section{Authors' contributions}

FBB designed the study, participated in data analysis and interpretation, and drafted the manuscript. DMG and PP participated in data interpretation and critically revised the manuscript for important intellectual content. All authors read and approved the final manuscript.

\section{Competing interests}

The authors declare that they have no competing interests.

\section{Consent for publication}

Not applicable.

\section{Ethics approval and consent to participate}

The study procedures were approved by the institutional review boards of all participating centres (https://adni.loni.usc.edu/wp-content/uploads/ how_to_apply/ADNI_Acknowledgement_List.pdf), and written informed consent was obtained from all participants or their authorised representatives. Ethics approval was obtained from the institutional review boards of each institution involved: Oregon Health and Science University; University of Southern California; University of California-San Diego; University of Michigan; Mayo Clinic, Rochester; Baylor College of Medicine; Columbia University Medical Center; Washington University, St. Louis; University of Alabama at Birmingham; Mount Sinai School of Medicine; Rush University Medical Center; Wien Center; Johns Hopkins University; New York University; Duke University Medical Center; University of Pennsylvania; University of Kentucky; University of Pittsburgh; University of Rochester Medical Center; University of California, Irvine; University of Texas Southwestern Medical School; Emory University; University of Kansas, Medical Center; University of California, Los Angeles; Mayo Clinic, Jacksonville;
Indiana University; Yale University School of Medicine; McGill University, Montreal-Jewish General Hospital; Sunnybrook Health Sciences, Ontario; U.B.C. Clinic for AD \& Related Disorders; Cognitive Neurology—St. Joseph's, Ontario; Cleveland Clinic Lou Ruvo Center for Brain Health; Northwestern University; Premiere Research Inst (Palm Beach Neurology); Georgetown University Medical Center; Brigham and Women's Hospital; Stanford University; Banner Sun Health Research Institute; Boston University; Howard University; Case Western Reserve University; University of California, Davis-Sacramento; Neurological Care of CNY; Parkwood Hospital; University of Wisconsin; University of California, Irvine-BIC; Banner Alzheimer's Institute; Dent Neurologic Institute; Ohio State University; Albany Medical College; Hartford Hospital, Olin Neuropsychiatry Research Center; Dartmouth-Hitchcock Medical Center; Wake Forest University Health Sciences; Rhode Island Hospital; Butler Hospital; UC San Francisco; Medical University South Carolina; St. Joseph's Health Care Nathan Kline Institute; University of lowa College of Medicine; Cornell University and University of South Florida: USF Health Byrd Alzheimer's Institute. The investigators within the ADNI contributed to the design and implementation of the ADNI and/or provided data but did not participate in analysis or writing of this report. A complete listing of ADNI investigators can be found online (http://adni.loni.usc.edu/wp-content/uploads/how_to_apply/ADNI_ Acknowledgement_List.pdf).

\section{Publisher's Note}

Springer Nature remains neutral with regard to jurisdictional claims in published maps and institutional affiliations.

\section{Author details}

${ }^{1}$ Toulouse Neurolmaging Centre (ToNIC), Université de Toulouse, Inserm/UPS, Toulouse, France. ${ }^{2}$ Nuclear Medicine Department, Purpan University Hospital, Toulouse, France. ${ }^{3}$ Nuclear Medicine Department, Lapeyronie University Hospital, Montpellier, France.

Received: 10 February 2017 Accepted: 24 March 2017

Published online: 26 April 2017

References

1. Petersen RC, Aisen P, Boeve BF, Geda YE, Ivnik RJ, Knopman DS, et al. Mild cognitive impairment due to Alzheimer disease in the community. Ann Neurol. 2013;74:199-208.

2. Ellendt S, Voß B, Kohn N, Wagels L, Goerlich K, Drexler E, et al. Predicting stability of mild cognitive impairment (MCI): findings of a community based sample. Curr Alzheimer Res. doi:10.2174/1567205014666161213120807.

3. Jack Jr CR, Knopman DS, Jagust WJ, Shaw LM, Aisen PS, Weiner MW, et al. Hypothetical model of dynamic biomarkers of the Alzheimer's pathological cascade. Lancet Neurol. 2010;9:119-28.

4. Sunderland T, Linker G, Mirza N, Putnam KT, Friedman DL, Kimmel LH, et al. Decreased $\beta$-amyloid ${ }_{1-42}$ and increased tau levels in cerebrospinal fluid of patients with Alzheimer disease. JAMA. 2003;289:2094-103.

5. Shaw LM, Vanderstichele H, Knapik-Czajka M, Clark CM, Aisen PS, Petersen RC, et al. Cerebrospinal fluid biomarker signature in Alzheimer's Disease Neuroimaging Initiative subjects. Ann Neurol. 2009;65:403-13.

6. Han SD, Gruhl J, Beckett L, Dodge HH, Stricker NH, Farias S, et al. Beta amyloid, tau, neuroimaging, and cognition: sequence modeling of biomarkers for Alzheimer's disease. Brain Imaging Behav. 2012;6:610-20.

7. Jack Jr CR, Wiste HJ, Vemuri P, Weigand SD, Senjem ML, Zeng G, et al. Brain beta-amyloid measures and magnetic resonance imaging atrophy both predict time-to-progression from mild cognitive impairment to Alzheimer's disease. Brain. 2010;133:3336-48.

8. Walhovd KB, Fjell AM, Brewer J, McEvoy LK, Fennema-Notestine C, Hagler DJ, et al. Combining MR imaging, positron-emission tomography, and CSF biomarkers in the diagnosis and prognosis of Alzheimer disease. AJNR Am J Neuroradiol. 2010;31:347-54.

9. Weigand SD, Vemuri P, Wiste HJ, Senjem ML, Pankratz VS, Aisen PS, et al. Transforming cerebrospinal fluid A $\beta 42$ measures into calculated Pittsburgh Compound B units of brain A $\beta$ amyloid. Alzheimers Dement. 2011;7:133-41.

10. Hansson $\mathrm{O}$, Zetterberg $\mathrm{H}$, Buchhave $\mathrm{P}$, Londos $\mathrm{E}$, Blennow K, Minthon L. Association between CSF biomarkers and incipient Alzheimer's disease in patients with mild cognitive impairment: a follow-up study. Lancet Neurol. 2006;5:228-34. 
11. Alexopoulos P, Werle L, Roesler J, Thierjung N, Gleixner LS, Yakushev I, et al. Conflicting cerebrospinal fluid biomarkers and progression to dementia due to Alzheimer's disease. Alzheimers Res Ther. 2016;8:51.

12. De Meyer G, Shapiro F, Vanderstichele H, Vanmechelen E, Engelborghs S, De Deyn PP, et al. Diagnosis-independent Alzheimer disease biomarker signature in cognitively normal elderly people. Arch Neurol. 2010;67:949-56

13. Visser PJ, Verhey F, Knol DL, Scheltens P, Wahlund LO, Freund-Levi Y, et al. Prevalence and prognostic value of CSF markers of Alzheimer's disease pathology in patients with subjective cognitive impairment or mild cognitive impairment in the DESCRIPA Study: a prospective cohort study. Lancet Neurol. 2009;8:619-27.

14. Pontecorvo MJ, Mintun MA. PET amyloid imaging as a tool for early diagnosis and identifying patients at risk for progression to Alzheimer's disease. Alzheimers Res Ther. 2011;3:11.

15. Clark CM, Pontecorvo MJ, Beach TG, Bedell BJ, Coleman RE, Doraiswamy PM, et al. Cerebral PET with florbetapir compared with neuropathology at autopsy for detection of neuritic amyloid- $\beta$ plaques: a prospective cohort study. Lancet Neurol. 2012;11:669-78.

16. Dubois B, Feldman HH, Jacova C, Hampel H, Molinuevo JL, Blennow K, et al. Advancing research diagnostic criteria for Alzheimer's disease: the IWG-2 criteria. Lancet Neurol. 2014;13:614-29.

17. Joshi AD, Pontecorvo MJ, Clark CM, Carpenter AP, Jennings DL, Sadowsky CH, et al. Performance characteristics of amyloid PET with florbetapir $F 18$ in patients with Alzheimer's disease and cognitively normal subjects. J Nucl Med. 2012;53:378-84.

18. Chen K, Roontiva A, Thiyyagura P, Lee W, Liu X, Ayutyanont N, et al. Improved power for characterizing longitudinal amyloid- $\beta$ PET changes and evaluating amyloid-modifying treatments with a cerebral white matter reference region. J Nucl Med. 2015;56:560-6.

19. Landau SM, Fero A, Baker SL, Koeppe R, Mintun M, Chen K, et al. Measurement of longitudinal $\beta$-amyloid change with ${ }^{18} \mathrm{~F}$-florbetapir PET and standardized uptake value ratios. J Nucl Med. 2015;56:567-74.

20. Brendel M, Högenauer M, Delker A, Sauerbeck J, Bartenstein P, Seibyl J, et al. Improved longitudinal $\left[{ }^{18} \mathrm{~F}\right]$-AV45 amyloid PET by white matter reference and VOI-based partial volume effect correction. Neuroimage. 2015;108:450-9.

21. Doraiswamy PM, Sperling RA, Johnson K, Reiman EM, Wong TZ, Sabbagh MN, et al. Florbetapir F 18 amyloid PET and 36-month cognitive decline: a prospective multicenter study. Mol Psychiatry. 2014;19:1044-51.

22. Landau SM, Mintun MA, Joshi AD, Koeppe RA, Petersen RC, Aisen PS, et al. Amyloid deposition, hypometabolism, and longitudinal cognitive decline. Ann Neurol. 2012;72:578-86.

23. Ong $K T$, Villemagne VL, Bahar-Fuchs A, Lamb F, Langdon N, Catafau AM, et al. $A \beta$ imaging with ${ }^{18} \mathrm{~F}$-florbetaben in prodromal Alzheimer's disease: a prospective outcome study. J Neurol Neurosurg Psychiatry. 2015;86:431-6.

24. Schreiber S, Landau SM, Fero A, Schreiber F, Jagust WJ. Alzheimer's Disease Neuroimaging Initiative. Comparison of visual and quantitative florbetapir F 18 positron emission tomography analysis in predicting mild cognitive impairment outcomes. JAMA Neurol. 2015;72:1183-90.

25. Ewers $M$, Insel P, Jagust WJ, Shaw L, Trojanowski JQ, Aisen P, et al. CSF biomarker and PIB-PET-derived beta-amyloid signature predicts metabolic, gray matter, and cognitive changes in nondemented subjects. Cereb Cortex. 2012;22:1993-2004.

26. Morris JC, Roe CM, Grant EA, Head D, Storandt M, Goate AM, et al. Pittsburgh compound B imaging and prediction of progression from cognitive normality to symptomatic Alzheimer disease. Arch Neurol. 2009;66:1469-75

27. Storandt M, Mintun MA, Head D, Morris JC. Cognitive decline and brain volume loss as signatures of cerebral amyloid-beta peptide deposition identified with Pittsburgh Compound B: cognitive decline associated with A $\beta$ deposition. Arch Neurol. 2009;66:1476-81.

28. Okello A, Koivunen J, Edison P, Archer HA, Turkheimer FE, Någren K, et al. Conversion of amyloid positive and negative $\mathrm{MCl}$ to $\mathrm{AD}$ over 3 years: an ${ }^{11}$ C-PIB PET study. Neurology. 2009;73:754-60.

29. Nordberg A, Carter SF, Rinne J, Drzezga A, Brooks DJ, Vandenberghe R, et al. A European multicentre PET study of fibrillary amyloid in Alzheimer's disease. Eur J Nucl Med Mol Imaging. 2013;40:104-14.

30. Boccardi M, Altomare D, Ferrari C, Festari C, Guerra UP, Paghera B, et al. Assessment of the incremental diagnostic value of florbetapir $F 18$ imaging in patients with cognitive impairment: the incremental diagnostic value of amyloid PET with $\left[{ }^{18} \mathrm{~F}\right]$-florbetapir (INDIA-FBP) Study. JAMA Neurol. 2016;73:1417-24.

31. Landau SM, Lu M, Joshi AD, Pontecorvo M, Mintun MA, Trojanowski JQ, et al. Comparing positron emission tomography imaging and cerebrospinal fluid measurements of $\beta$-amyloid. Ann Neurol. 2013;74:826-36.

32. Mattsson N, Insel P, Landau S, Jagust W, Donohue M, Shaw LM, et al. Diagnostic accuracy of CSF Ab42 and florbetapir PET for Alzheimer's disease. Ann Clin Transl Neurol. 2014;1:534-43.

33. Toledo JB, Bjerke M, Da X, Landau SM, Foster NL, Jagust W, et al. Nonlinear association between cerebrospinal fluid and florbetapir F-18 $\beta$-amyloid measures across the spectrum of Alzheimer disease. JAMA Neurol. 2015;72:571-81.

34. Shokouhi S, Mckay JW, Baker SL, Kang H, Brill AB, Gwirtsman HE, et al. Reference tissue normalization in longitudinal ${ }^{18} \mathrm{~F}$-florbetapir positron emission tomography of late mild cognitive impairment. Alzheimers Res Ther. 2016:8:2.

35. Weston PS, Paterson RW, Dickson J, Barnes A, Bomanji JB, Kayani I, et al. Diagnosing dementia in the clinical setting: can amyloid PET provide additional value over cerebrospinal fluid? J Alzheimers Dis. 2016:54:1297-302.

36. Hake A, Trzepacz PT, Wang S, Yu P, Case M, Hochstetler H, et al. Florbetapir positron emission tomography and cerebrospinal fluid biomarkers. Alzheimers Dement. 2015;11:986-93.

37. Palmqvist S, Zetterberg H, Blennow K, Vestberg S, Andreasson U, Brooks DJ, et al. Accuracy of brain amyloid detection in clinical practice using cerebrospinal fluid $\beta$-amyloid 42: a cross-validation study against amyloid positron emission tomography. JAMA Neurol. 2014;71:1282-9.

38. Palmqvist S, Mattsson N, Hansson O, Alzheimer's Disease Neuroimaging Initiative. Cerebrospinal fluid analysis detects cerebral amyloid- $\beta$ accumulation earlier than positron emission tomography. Brain 2016;139:1226-36

39. Mattsson N, Insel PS, Donohue M, Landau S, Jagust WJ, Shaw LM, et al. Independent information from cerebrospinal fluid amyloid- $\beta$ and florbetapir imaging in Alzheimer's disease. Brain. 2015;138:772-83.

40. Petersen RC, Aisen PS, Beckett LA, Donohue MC, Gamst AC, Harvey DJ, et al. Alzheimer's Disease Neuroimaging Initiative (ADNI): clinical characterization. Neurology. 2010;74:201-9.

41. McKhann G, Drachman DA, Folstein M, Katzman R, Price DL, Stadlan EM. Clinical diagnosis of Alzheimer's disease-report of the NINCDS-ADRDA Work Group under the auspices of Department of Health and Human Services Task Force on Alzheimer's Disease. Neurology. 1984;34:939-44.

42. Dubois B, Feldman HH, Jacova C, DeKosky ST, Barberger-Gateau P, Cummings J, et al. Research criteria for the diagnosis of Alzheimer's disease: revising the NINCDS-ADRDA criteria. Lancet Neurol. 2007;6:734-46.

43. Olsson A, Vanderstichele $H$, Andreasen $N$, De Meyer $G$, Wallin A Holmberg $B$, et al. Simultaneous measurement of $\beta$-amyloid ${ }_{1-42}$, total tau, and phosphorylated tau (Thr181) in cerebrospinal fluid by the xMAP technology. Clin Chem. 2005;51:336-45.

44. Landau SM, Breault C, Joshi AD, Pontecorvo M, Mathis CA, Jagust WJ, et al. Amyloid-beta imaging with Pittsburgh Compound B and florbetapir: comparing radiotracers and quantification methods. J Nucl Med. 2013;54:70-7.

45. Joshi A, Koeppe RA, Fessler JA. Reducing between scanner differences in multi-center PET studies. Neuroimage. 2009;46:154-9.

46. Resnick SM, Sojkova J. Amyloid imaging and memory change for prediction of cognitive impairment. Alzheimers Res Ther. 2011;3:3.

47. Fleisher AS, Chen K, Liu X, Roontiva A, Thiyyagura P, Ayutyanont N, et al. Using positron emission tomography and florbetapir $F 18$ to image cortical amyloid in patients with mild cognitive impairment or dementia due to Alzheimer disease. Arch Neurol. 2011;68:1404-11.

48. Aizenstein HJ, Nebes RD, Saxton JA, Price JC, Mathis CA, Tsopelas ND, et al. Frequent amyloid deposition without significant cognitive impairment among the elderly. Arch Neurol. 2008;65:1509-17.

49. Villemagne $V L$, Burnham S, Bourgeat $P$, Brown B, Ellis KA, Salvado O, et al. Amyloid $\beta$ deposition, neurodegeneration, and cognitive decline in sporadic Alzheimer's disease: a prospective cohort study. Lancet Neurol. 2013;12:357-67.

50. Gomar JJ, Conejero-Goldberg C, Davies P, Goldberg TE, Alzheimer's Disease Neuroimaging Initiative. Extension and refinement of the predictive value of 
different classes of markers in ADNI: four-year follow-up data. Alzheimers Dement. 2014;10:704-12.

51. Landau SM, Harvey D, Madison CM, Reiman EM, Foster NL, Aisen PS, et al. Comparing predictors of conversion and decline in mild cognitive impairment. Neurology. 2010;75:230-8.

52. Saint-Aubert L, Lemoine L, Chiotis K, Leuzy A, Rodriguez-Vieitez E, Nordberg A. Tau PET imaging: present and future directions. Mol Neurodegener. 2017;12:19.

53. Bennett DA, Schneider JA, Arvanitakis Z, Kelly JF, Aggarwal NT, Shah RC,

et al. Neuropathology of older persons without cognitive impairment from two community-based studies. Neurology. 2006;66:1837-44.

Submit your next manuscript to BioMed Central and we will help you at every step:

- We accept pre-submission inquiries

- Our selector tool helps you to find the most relevant journal

- We provide round the clock customer support

- Convenient online submission

- Thorough peer review

- Inclusion in PubMed and all major indexing services

- Maximum visibility for your research

Submit your manuscript at www.biomedcentral.com/submit 